\title{
South or Central American Indian
}

National Cancer Institute

\section{Source}

National Cancer Institute. South or Central American Indian. NCI Thesaurus. Code C77809.

Denotes a person having origins in one of the indigenous people of South or Central America such as Mayans or Incas. 